\title{
Czech Contribution to Athena
}

\author{
R. Hudec, L. Pína, V. Maršíková, A. Inneman, M. Skulinová, M. Míka
}

\begin{abstract}
We describe the recent status of the Czech contribution to the ESA Athena space mission, with emphasis on the development of new technologies and test samples of X-ray mirrors with precise surfaces, based on new materials, and their applications in space. In addition, alternative X-ray optical arrangements are investigated, such as Kirkpatrick-Baez systems.
\end{abstract}

\section{Introduction}

Design and development of X-ray optics have a long tradition in the Czech Republic (e.g. Hudec et al., 1991, 1999, 2000, 2001, Inneman et al. 1999, 2000). A range of related technologies have been exploited and investigated, including technologies for future large light-weight X-ray telescopes.

Future large space X-ray telescopes (such as Athena, considered by ESA [16]) require precise lightweight X-ray optics based on numerous thin reflecting shells. Novel approaches and advanced technologies need to be developed and exploited. In this paper, we refer to Czech efforts in connection with Athena, focusing on the results of test X-ray mirror shells produced by glass thermal forming (GTF) and by shaping $\mathrm{Si}$ wafers. Both glass foils and $\mathrm{Si}$ wafers are commercially available, have excellent surface microroughness of a few $0.1 \mathrm{~nm}$, and low weight (the volume density is $2.5 \mathrm{~g} \cdot \mathrm{cm}^{-3}$ for glass and $2.3 \mathrm{~g} \cdot \mathrm{cm}^{-3}$ for $\mathrm{Si}$ ). Technologies need to be exploited for shaping these substrates to achieve the required precise X-ray optic geometries without degrading the fine surface microroughness.

Although glass and, more recently, silicon wafers have been considered the most promising materials for future advanced large aperture X-ray telescopes, other alternative materials are also worth further study, such as amorphous metals and glassy carbon (Marsch et al., 1997). In order to achieve sub-arsec angular resolutions, the principles of active optics need to be adopted. The Athena X-ray observatory is a new X-ray telescope of the European Space Agency (ESA). This project supersedes IXO (International X-ray Observatory) as well as NASA's Constellation$\mathrm{X}$ concept and also ESA's XEUS mission concept (White et al., 2009). The spacecraft configuration for the Athena study is a mission featuring two large $\mathrm{X}$-ray telecopes, each with an optical bench with a focal length of approx. $11.5 \mathrm{~m}$ and a suite of focal plane instruments. The Athena mission concept was presented and discussed in detail at the 1st Athena science meeting held at MPE in Garching, Germany, in June 2011 [16].

\section{Czech contribution to Athena-related studies}

At the moment, the Czech contribution to Athena concentrates on: (1) participating in defining scientific goals, justification and project preparation, (2) participating in the design and development of mirror technologies. The first author of this paper was delegated as a member of the Athena Telescope Working Group. In mirror development, we focus on supporting ESA ESTEC micropore silicon technology design and also on designing and developing alternative background technologies and designs, as discussed in greater detail below. Originally, these technologies were studied for the ESA/NASA/JAXA IXO project, but we believe that at least some of them might be valuable for Athena.

\section{The glass foil option}

Glass science and technology has a long tradition in the Czech Republic. At the same time, glass technology is one of the most promising technologies for producing mirrors for Athena and/or similar space telescopes, as the volume density of glass is more than 3 times less than the volume density of electroformed nickel layers. Glass foils can be used as flats, or may be shaped or thermally slumped to achieve the required geometry. Thermal forming of glass is not a new technology. It has been used in various sectors of the glass industry and in glass art, as well as in the production of Cherenkov mirrors. However, the application of this technology in X-ray optics is related to the need to improve accuracy significantly and minimize errors. As the first step, small (various sizes typically less than $100 \times 100 \mathrm{~mm}$ ) glass samples of various types provided by various manufacturers were used and thermally shaped. The geometry 
was either flat or curved (cylindrical or parabolic). The project continued with larger samples (up to $300 \times 300 \mathrm{~mm}$ ) and further profiles. Recent efforts have focused on optimizing the relevant parameters of both glass material and substrates, as well as the parameters of the slumping process.

Various approaches have been investigated. We note that these are not quite identical with efforts by other teams (e.g. Zhang et al., 2010, Ghigo et al., 2010). The glass samples were thermally formed at Rigaku, Prague, and also at the Institute of Chemical Technology in Prague. For large samples $(300 \times 300 \mathrm{~mm})$, facilities at the Optical Development Workshop in Turnov were used. The strategy is to develop a technology suitable for inexpensive mass production of thin X-ray optics shells, i.e., to avoid expensive mandrels and techniques that are not suitable for mass production, or that are too expensive. Numerous glass samples have been shaped and tested in order to find the optimal parameters. The shapes and profiles of both mandrels, as well as the resulting glass replicas, have been carefully measured using metrological devices. The results show that the quality of the thermal glass replica can be significantly improved by optimizing the material and improving the design of the mandrel, by modifying the thermal forming process, as well as by optimizing the temperature. After the modifications and improvements, some of them significant, we obtained the resulting deviation of the thermally formed glass foil from the ideal designed profile to be less than $1 \mu \mathrm{m}$ (peak to valley value) in the best case. This value is, however, strongly dependent on the exact temperature, so we believe that further improvements are still possible.

The fine original microroughness (typically better than $1 \mathrm{~nm}$ ) of the original float glass foil was found not to be degraded by the thermal forming process. We note that our approach in thermal glass forming is different from the approaches used by other authors. Recent efforts have been devoted to optimizing the whole process, using and comparing different forming strategies etc., as the final goal is to further improve the forming accuracy to less than $0.1 \mu \mathrm{m}$ values. For the near future, we plan to continue these efforts together with investigations of computer-controlled forming of glass foils (according to the principles of active optics).

\section{The silicon wafer option}

Silicon is a relatively light material and already during the manufacturing process it is lapped and polished (either on one side or on both sides) to very fine smoothness (better than a few $0.1 \mathrm{~nm}$ ) and thickness homogeneity (of the order of $1 \mu \mathrm{m}$ ). Another obvious option, recently considered as one of most promising for high-precision X-ray optics for Athena, is the use of X-ray optics based on commercially available silicon wafers manufactured mainly for the purposes of the semiconductor industry.

The main advantages of the application of $\mathrm{Si}$ wafers in space X-ray optics are (i) the volume density, which is more than 3 times lower than the electroformed nickel used in the past for galvanoplastic replication of multiply nested X-ray mirrors, and slightly less than the alternative approach of glass foils, (ii) very high thickness homogeneity, typically less than $1 \mu \mathrm{m}$ over $100 \mathrm{~mm}$, and (iii) very small surface microroughness either on one side or on both sides (typically of the order of a few $0.1 \mathrm{~nm}$ or even less). Silicon wafers were expected to be used in the ESA XEUS and IXO projects, and are still under consideration for the Athena project. The recent baseline optics for the Athena X-ray telescope design is, like XEUS and IXO, based on X-Ray High Precision Pore Optics (X-HPO), a technology currently under development with ESA funding (RDOpt, RD-HPO), with a view to achieving large effective areas with low mass, reduced telescope length, high stiffness, and a monolithic structure, favoured for handling the thermal environment and for simplifying the alignment process (Bavdaz et al. 2010). In addition, due to the higher packing density and the associated shorter mirrors required, conical approximation to the Wolter-I geometry becomes possible. $\mathrm{X}$-HPO optics is based on ribbed $\mathrm{Si}$ wafers stacked together. The Si wafers for achieving the conical approximation are formed by stacking a large number of plates together using a mandrel. The typical size of the Si wafers is $10 \times 10 \mathrm{~cm}$.

There are also alternative X-ray optics arrangements with the use of Si wafers. In this paper, we refer to the development of an alternative design of innovative precise X-ray optics based on Si wafers. Our approach is based on two steps, namely (i) developing dedicated $\mathrm{Si}$ wafers with properties optimized for use in space X-ray telescopes, and (ii) precisely shaping the wafers to the optical surfaces (Figure 4). Stacking to achieve nested arrays is performed after the wafers have been shaped. In this approach, Multi Foil Optics (MFO) is thus created from shaped $\mathrm{Si}$ wafers. For more details on MFO, see Hudec et al. (2005).

This alternative approach does not require the $\mathrm{Si}$ wafers to have a ribbed surface, so problems with transferring any deviation, stress, and/or inaccuracy from one wafer to the neighbouring plates or even to the whole stacked assembly will be avoided. However, suitable technologies for precise stacking of optically formed wafers to a multiple array have to be developed.

The Si wafers available on the market are designed for use mainly in the semiconductor industry. It is obvious that the requirements of this industry 
are not the same as the requirements of precise space X-ray optics. Si wafers are a monocrystal (single crystal) with some specifics, and this must also be taken into account. Moreover, Si wafers are fragile, and it is very difficult to bend and/or shape them precisely for the thicknesses required for X-ray telescopes, i.e. around $0.3-1.0 \mathrm{~mm}$. An exception is thin $\mathrm{Si}$ wafers below $0.1 \mathrm{~mm}$ in thickness. However, these can be hardly used in this type of X-ray optics because of diffraction limits. Also, while their thickness homogeneity is mostly perfect, the same is not true in the case of commercially available wafers for their flatness (note that we refer here to the deviation of the upper surface of a free-standing Si wafer from the ideal plane, while in the semiconductor community flatness is usually represented by a set of parameters).

In order to achieve the very high accuracy required by ESA for future large space X-ray telescopes like Athena, the parameters of the Si wafers need to be optimized (for application in X-ray optics) at the production stage. For this purpose we have established and developed a multidisciplinary working group including specialists from the development department of the $\mathrm{Si}$ wafer industry with the goal to design and manufacture Si wafers with improved parameters (mostly flatness) optimized for application in X-ray telescopes. It should be noted that the manufacture of silicon wafers is a complicated process with numerous technological steps and with many free parameters that can be modified and optimized to achieve optimal performance. This can also be useful for further improving the quality of $\mathrm{X}-\mathrm{HPO}$ optics. As we are dealing with high-quality $\mathrm{X}$-ray imaging, the smoothness of the reflecting surface is important. The standard microroughness of commercially available Si wafers (we have used the products of ON Semiconductor, Czech Republic) is of the order of $0.1 \mathrm{~nm}$, as confirmed by several inde- pendent measurements using various techniques including the Atomic Force Microscope (AFM). This is related to the method of chemical polishing used in the manufacture of Si wafers. The microroughness of $\mathrm{Si}$ wafers exceeds the microroughness of glass foils and most other alternative mirror materials and substrates. The flatness (in the sense of the deviation of the upper surface of a free-standing $\mathrm{Si}$ wafer from a plane) of commercially available Si wafers was however found not to be optimal for use in high-quality (order of arcsec angular resolutions) X-ray optics. Most $\mathrm{Si}$ wafers show deviations from the plane of the order of a few tens of microns. After modifying the technological process during Si wafer manufacture, we were able to reduce this value to just a few microns. Also, the thickness homogeneity was improved. In collaboration with the manufacturer, further steps are planned to improve the flatness (deviation from an ideal plane) and the thickness homogeneity of $\mathrm{Si}$ wafers. These and planned improvements introduced at the Si wafer manufacture stage can also be applied for other designs of $\mathrm{Si}$ wafer optics including X-HPO, and can play a crucial role in the Athena project.

The X-ray optics design for Athena is based on the Wolter 1 arrangement, and hence requires curved surfaces. However, due to the material properties of monocrystalline $\mathrm{Si}, \mathrm{Si}$ wafers (except very thin ones) are extremely difficult to shape. It is obvious that we have to overcome this problem in order to achieve the fine accuracy and stability required for future large $\mathrm{X}$-ray telescopes. The final goal is to provide optically shaped Si wafers with no or little internal stress. Three different alternative technologies for shaping Si wafers have been designed and tested to achieve precise optical surfaces (Figure 1). The samples shaped and tested were typically 100 to $150 \mathrm{~mm}$ large, typically 0.6 to $1.3 \mathrm{~mm}$ thick, and were bent to either cylindrical or parabolic test surfaces.
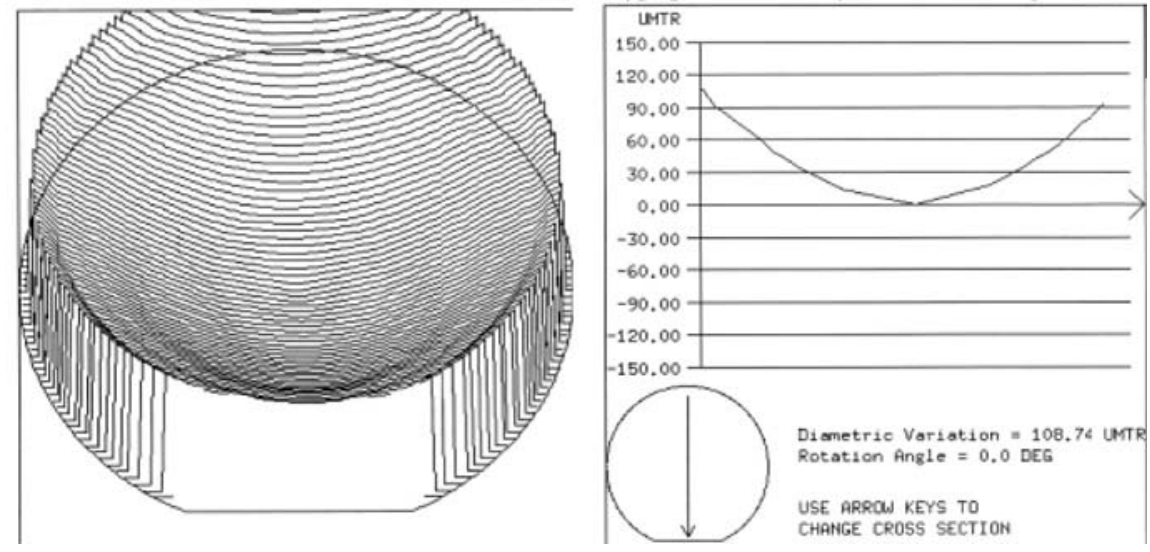

Fig. 1: Example of precise silicon wafer shaping. After deposition of poly-Si (thickness $1436 \mathrm{~nm}$ at temperature $615^{\circ} \mathrm{C}$ ) and for wafer thickness $507 \mu \mathrm{m}$, a warp of $110 \mathrm{~mm}(R=25.6 \mathrm{~m})$ was achieved. Left: Wafer deformation map. Right: Warp profile perpendicular to the facet 
The development described here is based on a scientific approach, and hence the large number of samples formed with different parameters must be precisely measured and investigated in detail. Especially precise metrology and measurements play a crucial role in this type of experiment. The samples of bent wafers with the investigated technologies have been measured, including Taylor-Hobson mechanical and STILL optical profilometry, as well as optical interferometry (ZYGO) and AFM (Atomic Force Miscroscope) analyses. It has been confirmed that all these three technologies do not degrade the intrinsic fine microroughness of the wafer. While the two physical/chemical technologies exploited give peakto-valley (PV) deviations (of the real surface of the sample compared with the ideal optical surface) of less than 1 to $2 \mu \mathrm{m}$ over the $150 \mathrm{~mm}$ sample length as preliminary values, the deviations of the first thermally bent sample are larger, of the order of $10 \mu \mathrm{m}$. Taking into account that the applied temperatures, as well as other parameters, were not optimized for this first sample, we anticipate that the PV (peak to valley) value can be further reduced down to the order of $1 \mu \mathrm{m}$ and perhaps even below. Fine adjustments of the parameters can also further improve the accuracy of the results for the other two techniques.

\section{The Kirkpatrick-Baez option}

Although Wolter systems are generally well-known and widely used, Hans Wolter was not the first to propose X-ray imaging systems based on reflection of X-rays. In fact, the first grazing incidence system to form a real image was proposed by Kirkpatrick and Baez in 1948. This system consists of a set of two orthogonal parabolas of translation (Figure 2). The first reflection focuses to a line, which is focused by the second surface to a point. This was necessary to avoid the extreme astigmatism suffered by a single mirror, but it still was not free of geometric aberrations. Nevertheless, the system is attractive because it is easy to construct the reflecting surfaces. These surfaces can be produced as flat plates and then mechanically bent to the required curvature. In order to increase the aperture, a number of mirrors can be nested together, but it should be noted that this nesting introduces additional aberrations. This configuration is used mostly in experiments not requiring a large collecting area (solar, laboratory). The applications in X-ray astronomy and astrophysics were limited in the past, despite initial success on sounding rockets (e.g. Gorenstein et al., 1978). Nevertheless, large modules of Kirkpatrick-Baez (KB) mirrors based on float glass have also been suggested for stellar X-ray experiments (LAMAR experiment in KB configuration designed for the Shuttle experiment, e.g. Fabricant et al. 1988).

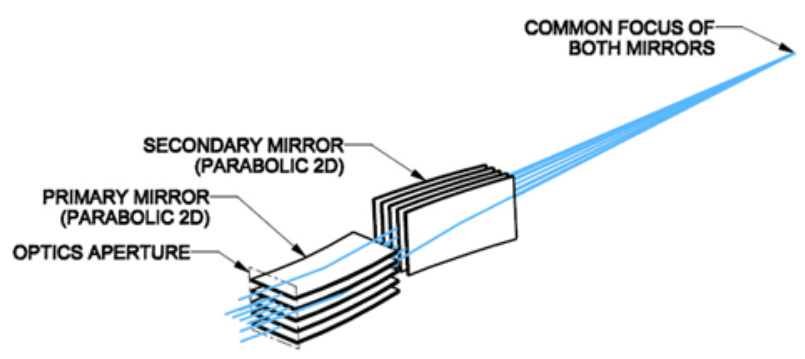

Fig. 2: Multi Foil Optics (MFO) in the Kirkpatrick-Baez (K-B) arrangement

Superior silicon substrates have recently become available that make it possible to consider designing large $\mathrm{KB}$ modules with this novel material. As mentioned above, Si wafers are difficult to shape, especially to small radii. To overcome this difficulty, another X-ray optics arrangement can be considered, namely the Kirkpatrick-Baez (KB) system. Then the curvature radii are much larger, of the order of a few $\mathrm{km}$, while the imaging performance is similar. For the same effective area, however, the focal length of the $\mathrm{KB}$ system is about twice as large as the focal length of the Wolter system. Nevertheless, KB systems represent a promising alternative to the classical Wolter systems in future large space X-ray telescopes.

A very important factor is the ease (and hence the reduced cost) of constructing highly segmented modules based on multiply nested thin reflecting substrates, in comparison with the Wolter design. While e.g. the Wolter design for Athena requires the substrates to be precisely formed with curvatures as small as $0.25 \mathrm{~m}$, the alternative KB arrangement uses almost flat or only slightly bent sheets. Hence the feasibility of constructing a KB module with the required 5 arcsec FWHM at an affordable cost is higher than for the Wolter arrangement.

Advanced KB telescopes are based on the Multi Foil Optics (MFO) approach (X-ray grazing incidence imaging optics based on numerous thin reflecting substrates/foils). The distinction between MFO and other optics using packed or nested mirrors is that MFO is based on numerous very thin (typically less than $0.1 \mathrm{~mm}$ ) substrates. The MFO KB test modules were recently designed and constructed at Rigaku Innovative Technologies Europe (RITE) in Prague, and 2 modules were tested in full aperture X-ray tests in the test facility of the University of Boulder, with preliminary results of FWHM 26 arcsec for a full stack of 24 standard Si plates at $5 \mathrm{keV}$, and even better for glass foils and 1D imaging (Figures 3,4). 

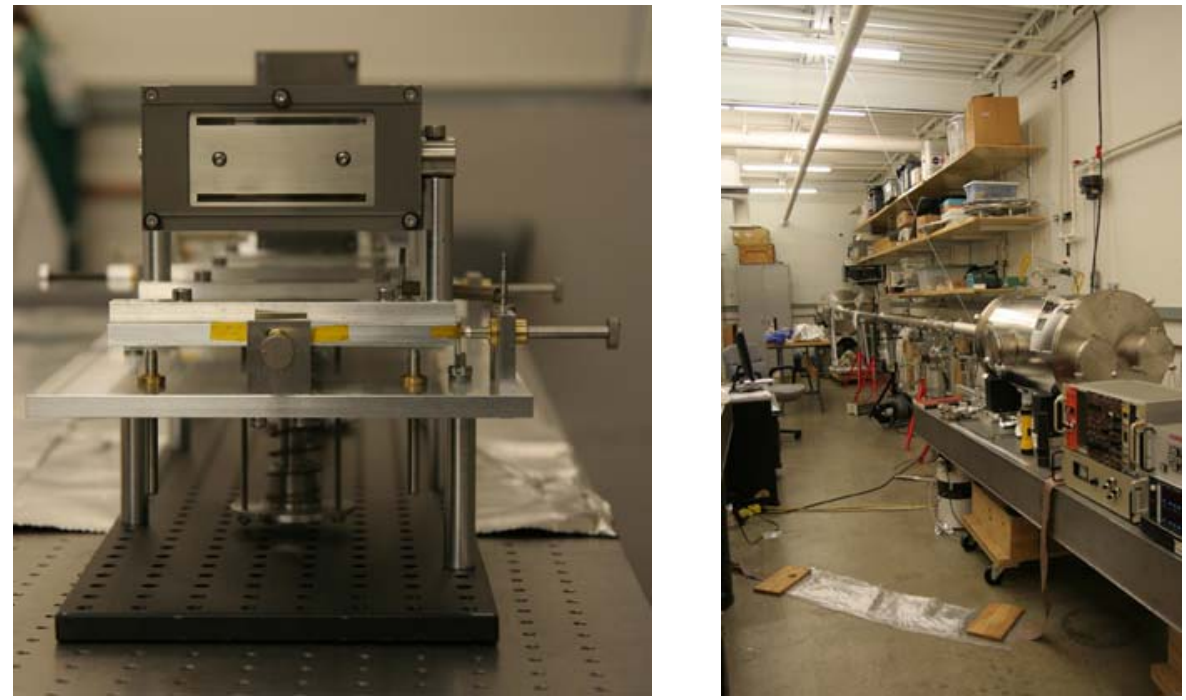

Fig. 3: Left: Test K-B modules assembled at Rigaku RITE in Prague, Right: The X-ray test facility for full aperture $\mathrm{X}$-ray tests at the University of Colorado in Boulder
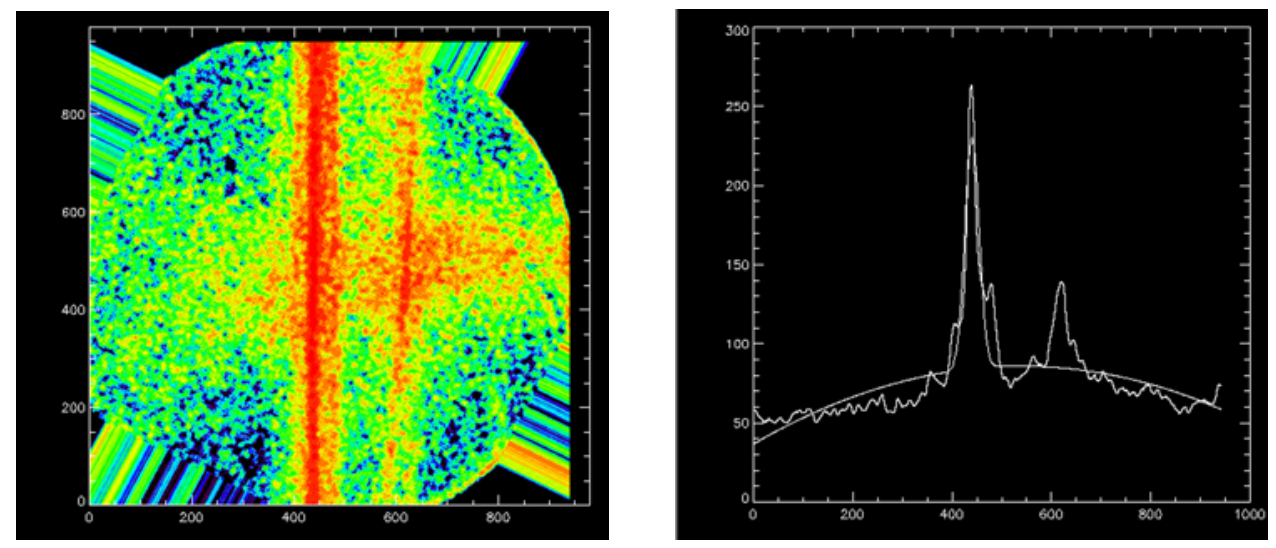

Fig. 4: The measurement results of the K-B 1D test module with glass foils, full aperture tests at 5 keV at the University of Colorado at Boulder. The estimated FWHM of 1D focus is 4 arcsec

In our opinion, the use of KB design instead of Wolter in Athena might help to retain high performance (such as effective area) even in the event of a reduced budget for Athena.

\section{Conclusion}

Suitable technologies for future large X-ray telescopes require extensive research work. Two promising technologies suitable for future large-aperture and fine resolution X-ray telescopes, such as Athena, were exploited and investigated in detail, namely Glass Thermal Forming and Si wafer bending. In both cases, promising results have been achieved, with peak-to-valley deviations of the final profiles from the ideal profiles being of the order of $1 \mu \mathrm{m}$ in the best cases, with space for further essential improve- ments and optimization. In the Czech Republic, an interdisciplinary team with 10 members is cooperating closely with experienced specialists, including researchers from a large company producing Si wafers. $\mathrm{Si}$ wafers have been successfully bent to the desired geometry by three different techniques. In the best cases, the accuracy achieved for a $150 \mathrm{~mm}$ Si wafer is 1-2 $\mu \mathrm{m}$ for deviation from the ideal optical surface. Experiments are continuing in an attempt to further improve the forming accuracy.

As an alternative, we have investigated the $\mathrm{KB}$ option for Athena, with quite promising results justifying further efforts in this direction. A major advantage of $\mathrm{KB}$ is its low cost, as there is no need for bending to small curvatures and/or for the use of (expensive) mandrels. Less expensive optics can maintain a high effective area even on a reduced budget. 


\section{Acknowledgement}

We acknowledge the support provided by the Grant Agency of the Academy of Sciences of the Czech Republic, grant IAAX 01220701, by the Ministry of Education and Youth of the Czech Republic, projects ME918, ME09028 and ME09004. The investigations related to the ESA IXO (now Athena) project were supported by ESA PECS Project No. 98038. We also acknowledge collaboration with Drs. J. Sik and M. Lorenc from ON Semiconductor Czech Republic, and with the team of Prof. Webster Cash from the University of Colorado at Boulder for X-ray tests of $\mathrm{KB}$ modules in their X-ray facility.

\section{References}

[1] Hudec, R., Valnicek, B., Cervencl, J., et al.: SPIE, 1 343, 162, 1991.

[2] Hudec, R., Pina, L., Inneman, A.: SPIE, 3766 , 62, 1999.

[3] Hudec, R., Pina, L., Inneman, A.: SPIE, 4012 , 422, 2000.

[4] Hudec, R., Inneman, A., Pina, L.: In LobsterEye: Novel X-ray Telescopes for the 21st Century, New Century of X-ray Astronomy, ASP Conf. Proc., 251, 542, 2001.

[5] Hudec, R., Pina, L., Inneman, A. et al.: SPIE, $5 \mathbf{9 0 0 ,} 276,2005$.

[6] Inneman, A., Hudec, R., Pina, L., Gorenstein, P.: SPIE, $\mathbf{3}$ 766, 72, 1999.

[7] Inneman, A., Hudec, R., Pina, L.: SPIE, $4 \mathbf{1 3 8}$ 94, 2000.

[8] Kirkpatrick, P., Baez, A. V.: J. Opt. Soc. Am. 38, 766, 1948.

[9] Marsch, H., et al.: Introduction to Carbon Technologies, University of Alicante, 1997.

[10] White, N. E., Hornschemeier, A. E.: Bulletin of the American Astronomical Society, Vol. 41, 2009 , p. 388.

[11] White, N. E., Parmar, A., Kunieda, H.: International X-ray Observatory Team, 2009, Bulletin of the American Astronomical Society, Vol. 41, p. 357 .

[12] http://ixo.gsfc.nasa.gov

[13] Bavdaz, M., et al.: Proceedings of the SPIE, Vol. 7 732, 2010, p. 77321E-77321E-9.

[14] Zhang, W. W., et al.: Proceedings of the SPIE, Vol. 7 732, 2010, p. 77321G-77321G-8.

[15] Ghigo, M., et al.: Proceedings of the SPIE, Vol. 7 732, 2010, p. 77320C-77320C-12.

[16] http://www.mpe.mpg.de/athena/ home.php?lang=en

[17] Gorenstein, P., et al.: Astrophys. J. 224, 718, 1978.

[18] Fabricant, D. G., et al.: Applied Optics, 27, No. 8, $1456,1988$.

René Hudec

E-mail: rhudec@asu.cas.cz

Astronomical Institute

Academy of Sciences of the Czech Republic

25165 Ondřejov, Czech Republic

Faculty of Electrical Engineering

Czech Technical University in Prague

Technická 2, 16627 Prague, Czech Republic

Ladislav Pína

Faculty of Nuclear Engineering

Czech Technical University in Prague

Břehová 78/7, 11000 Prague, Czech Republic

Veronika Maršíková

Adolf Inneman

Rigaku Innovative Technologies Europe, s. r. o.

Novodvorská 994, 142 21, Prague 4, Czech Republic

Michaela Skulinová

Astronomical Institute

Academy of Sciences of the Czech Republic

25165 Ondřejov, Czech Republic

Martin Míka

Institute of Chemical Technology

Technická 5, 16628 Prague, Czech Republic 